http://dx.doi.org/10.18778/2196-8403.2007.02

\title{
Zum Schwerpunkt
}

Der thematische Schwerpunkt dieser Convivium-Ausgabe ist den ,gender studies' gewidmet, die in den 90er Jahren des 20. Jahrhunderts als transdisziplinäre Studiengänge an einigen deutschen und polnischen Universitäten eingerichtet wurden. In Westdeutschland konnten sie auf die bereits seit den 80er Jahren etablierte Frauenforschung (,women's studies") aufbauen. In Polen hingegen fiel die Entstehung der Frauen- und dann auch der Geschlechterforschung in den demokratischen Aufbruch nach 1989; sie sind ein Teil von ihm und ohne ihn nicht zu denken. Mit dem Kampf um Chancengleichheit und Selbstbestimmung von Frauen unmittelbar verbunden, sind ,gender studies ' in Polen stärker als in Deutschland politisiert, wo die Frauenforschung bereits Anfang der 90er Jahre einen fast ausschließlichen akademischen Charakter angenommen hatte.

Die polnische Germanistik nähert sich vorsichtig der Frauen- und Geschlechterforschung. Wir sind daher erfreut, daß so viele Aufsätze im SchwerpunktTeil dieser Convivium-Ausgabe veröffentlicht werden können. Einleitend befaßt sich Bożena Chołuj mit dem Thema ,gender studies‘ und Literaturwissenschaft. Die Autorin - Mitbegründerin der ,gender studies‘ an der Warschauer Universität im Jahr 1996 - gibt eine Einführung in die Entwicklung der Geschlechterforschung und betont das Innovatorische, das das Operieren mit der Kategorie ,gender' gegenüber der Kategorie ,Frau' und damit der ,gender studies‘ gegenüber den ,women's studies‘ erbringt. Nach Judith Butler sei ,gender' „eine der wichtigsten Kategorien [...], mit der der biologistische Geschlechterdiskurs, auf den sich die meisten Traditionen stützen, überwunden werden könne." (S. 15) Eine Untersuchung von Konstruktion und Funktion der Kategorie ,Geschlecht" auf verschiedenen kulturellen Ebenen - in den Wissenschaften, auf der symbolischen Ebene und auf der Ebene kultureller Praktiken und Normen - ermögliche es, „Geschlecht als veränderbar“ und die „Geschlechterbeziehungen gerechter" zu denken. (S. 15) Chołuj hebt die politische Relevanz der ,gender studies" hervor und verweist auf europäische genderpolitische Initiativen wie die , gender-sensitive Education`. Literatur kann in dieser Hinsicht als eine von diskursiven Praktiken beschrieben werden, welche die Geschlechterdifferenz reproduzieren und stabilisieren oder spielerisch, subversiv unterlaufen. Die Analyse dieser Opposition ist somit eine wichtige Aufgabe der Literaturwissenschaft. Besondere Aufmerksamkeit verdient Chołujs Darstellung der polnischen ,gender studies', deren Entstehungszusammenhang sie von der Zeit der Solidarność über die Transformationszusam- 
menhänge hinweg umreißt. Wesentliche Merkmale der zunächst in Warschau und Krakau entstandenen , gender studies' sind demnach:

- die anfängliche Konzentration auf Analysen von Diskriminierungspraktiken und Machtstrukturen am Beispiel politischer Reden, rechtlicher und literarischer Texte sowie der Medien;

- die Bedeutung außerakademischer Kreise, im Besonderen der NGOs, für die Entstehung der , gender studies';

- die Orientierung an Begriffen und der Arbeitsweise der Polonistin und Romantik-Forscherin Maria Janion;

- das Untersuchen von Ausschluß- und Diskriminierungsmechanismen im nationalen Diskurs in ihrer Verschränkung von Ethnie und Geschlecht (z.B. von judenfeindlichen Darstellungen in bekannten literarischen Texten);

- die besondere Bedeutung der Literaturwissenschaft und hier der - ein Novum darstellenden - Zusammenarbeit zwischen den Polonistinnen und Neuphilologinnen;

- die Gleichzeitigkeit von Forschungsansätzen, die sich in der westlichen Frauen- und Geschlechterforschung nacheinander herausgebildet haben, dabei auch die Parallelität von Frauen und Geschlechterforschung.

Gabriela Brudzyńska-Němec' Artikel ist ein Beitrag zur Geschichte der Frauenemanzipation in Deutschland, in der die deutsch-polnische Perspektive kaum eine Rolle spielt. Sie zeigt, daß damit Wichtiges verloren geht. Die nach der Niederschlagung des polnischen Novemberaufstandes 1830/31 in Deutschland entstandenen Polenvereine können als die ersten legal tätigen politischen Organisationen angesehen werden, die einen politischen Wandel anstrebten. Zweifellos hat die Unterstützung der polnischen Aufständischen und dann der durch weite Teile Deutschlands, insbesondere Süddeutschlands ziehenden Emigranten, die politischen Anschauungen der Liberalen radikalisiert. Brudzyńska-Němec, die seit Jahren zur Tätigkeit der deutschen Polenvereine in Süddeutschland arbeitet, untersucht in ihrem Beitrag, welchen Anteil die Frauen an der liberalen Polenhilfe nahmen, indem sie zeitgenössische Dokumente hinsichtlich deren Teilnahme am Hambacher Fest und der weiblichen Polenvereine auswertet. Die Aufgabe letzterer beschränkte sich keineswegs auf Wohltätigkeit, sondern kann als ,tätige Anteilnahme“ am polnischen Unabhängigkeitskampf verstanden werden. Die Polenhilfe stellt damit „ein Bindeglied zwischen den Aktivitäten der Frauen zur Zeit der Befreiungskriege und 
zur Zeit der Revolution 1848“ dar (S. 41) und sollte als eine wichtige Phase in der Geschichte der deutschen Frauenemanzipation gewertet werden.

Im folgenden Beitrag analysiert Andreas Degen die Gestaltung der polnischen Wanda-Figur in der deutschsprachigen Literatur des 19. Jahrhunderts und fragt nach dem dabei konstruierten Frauenbild. An literarischen Werken von Friedrich Schiller bis C. Jürgens zeigt er, wie die mit der legendären Wanda-Figur verbundene sittliche Überlegenheit allmählich in eine erotische transformiert und die Figur zu einer Projektionsfläche außerbürgerlicher Weiblichkeit wird. Schließlich wird auch diese Überlegenheit zunehmend unterhöhlt, so daß die Wanda-Figur nur noch „die Position einer mehr oder weniger passiven Erfüllungsgehilfin männlichen Begehrens einnimmt" (S. 76). Stellt der Aufsatz von Degen einen Beitrag zur Erforschung von Weiblichkeitsmustern in der Literatur dar, so untersucht Justyna Górny am Beispiel der frühen Rezeption der Werke von Franziska zu Reventlow, in welcher Weise die Konstruktionen des Autor- und Werkbegriffes durch die Kategorie ,Geschlecht' geprägt sein können. In einer eingehenden Analyse der Rezensionen weist sie nach, daß die Wahrnehmung des Werkes von Reventlow mehr und mehr an die Biographie und das Geschlecht seiner Autorin gebunden wurde. Während die Rezensenten des Romandebüts Ellen Olestjerne noch das Universelle der erzählten Lebensgeschichte hervorhoben, werden spätere Lektüren zu fiktiven Begegnungen mit der Autorin Franziska zu Reventlow. Die Literarizität des Textes geht bei einer solchen Sichtweise verloren.

Monika Szczepaniak, die sich bereits in ihrer Habilitationsschrift zum Blaubart-Motiv in der deutschen Literatur mit Männlichkeitsbildern beschäftigt hat, vergleicht in ihrem Aufsatz die Konstruktion deutscher und polnischer Männlichkeit anhand von Texten zum Ersten Weltkrieg. Dabei arbeitet sie trotz der Feststellung einiger Gemeinsamkeiten - die Indienstnahme durch die nationale Idee, die Kreierung der Einzelkämpfer-Mythen des Fliegers und des Kavalleristen - einen wesentlichen Unterschied heraus: Die polnische Konstruktion militärischer Männlichkeit scheint Weiblichkeit integrieren zu können, während die deutsche einen , harten' Männlichkeitstypus darstellt.

Der letzte Beitrag ist der Schriftstellerin Helga M. Novak gewidmet, die seit längerem in Polen lebt, deren Werk aber immer noch der Übersetzung ins Polnische harrt. Izabela Surynt untersucht eines seiner zentralen Themen: das der Gewalt. Diese wird im Werk Novaks in verschiedenen Formen vorgeführt, als Gewalt in der Familie, in den Geschlechterbeziehungen, als institutionelle Gewalt, wie sie unterschiedlichen politischen Systemen eigen ist, wobei jedoch insbesondere der sozialistische Staat ins Visier genommen wird. An weibli- 


\section{Zum Schwerpunkt}

chen Figuren läßt die Dichterin häufig verschiedene Arten der Gewalt mit der Diskriminierung der kulturell ,Fremden“ konvergieren. Obwohl die bis hin zur Autoaggression reichende Zerstörungsmacht von Gewalt eindrücklich gezeigt wird, sind Novaks Figuren keine Opfer. Sie behaupten sich und brechen aus den Gewaltszenarien immer wieder aus, die auch „,neue identitätsstiftende Energien“ freilegen (S. 122). Abschließend sei noch auf die Buchbesprechungen zum Schwerpunktthema hingewiesen, die sich im Rezensionsteil befinden. Und es sei hier der Wunsch ausgesprochen, daß es Convivium mit den diesjährigen Schwerpunktbeiträgen gelingen möge, zu weiterführenden Forschungen anzuregen.

Marion Brandt 